\title{
Artificial Induction of Associative Olfactory Memory by Optogenetic and Thermogenetic Activation of Olfactory Sensory Neurons and Octopaminergic Neurons in Drosophila Larvae
}

OPEN ACCESS

Edited by:

Martin Giurfa,

Université Paul Sabatier-Toulouse III,

France

Reviewed by:

Liria Monica Masuda-Nakagawa,

University of Cambridge, UK

Ayse Yarali,

Leibniz Institute for Neurobiology,

Germany

*Correspondence:

Takato Honda

s1330540@u.tsukuba.ac.jp:

Katsuo Furukubo-Tokunaga

furukubo-tokunaga.gm@

u.tsukuba.ac.jp

Received: 11 December 2015

Accepted: 15 June 2016

Published: 28 June 2016

Citation:

Honda T, Lee C-Y, Honjo K and Furukubo-Tokunaga K (2016) Artificial Induction of Associative Olfactory

Memory by Optogenetic and

Thermogenetic Activation of Olfactory Sensory Neurons and Octopaminergic

Neurons in Drosophila Larvae. Front. Behav. Neurosci. 10:137. doi: 10.3389/fnbeh.2016.00137

\author{
Takato Honda ${ }^{1,2,3 *}$, Chi-Yu Lee ${ }^{1,3}$, Ken Honjo ${ }^{1}$ and Katsuo Furukubo-Tokunaga ${ }^{1 *}$ \\ ${ }^{1}$ Institute of Biological Sciences, University of Tsukuba, Tsukuba, Japan, ${ }^{2}$ Ph.D. Program in Human Biology, School of \\ Integrative and Global Majors, University of Tsukuba, Tsukuba, Japan, ${ }^{3}$ International Institute for Integrative Sleep Medicine \\ (WPI-IIIS), University of Tsukuba, Tsukuba, Japan
}

The larval brain of Drosophila melanogaster provides an excellent system for the study of the neurocircuitry mechanism of memory. Recent development of neurogenetic techniques in fruit flies enables manipulations of neuronal activities in freely behaving animals. This protocol describes detailed steps for artificial induction of olfactory associative memory in Drosophila larvae. In this protocol, the natural reward signal is substituted by thermogenetic activation of octopaminergic neurons in the brain. In parallel, the odor signal is substituted by optogenetic activation of a specific class of olfactory receptor neurons. Association of reward and odor stimuli is achieved with the concomitant application of blue light and heat that leads to activation of both sets of neurons in living transgenic larvae. Given its operational simplicity and robustness, this method could be utilized to further our knowledge on the neurocircuitry mechanism of memory in the fly brain.

Keywords: Drosophila, learning and memory, behavior assay, optogenetics, thermogenetics

\section{INTRODUCTION}

In the past decades, the development of powerful neurogenetic techniques in the fruit fly Drosophila melanogaster has contributed to further studies on the molecular and cellular mechanisms of learning and memory (Heisenberg, 2003; Davis, 2011; Guven-Ozkan and Davis, 2014). In particular, with simple and identifiable neural networks, Drosophila larvae provide an ideal system for the elucidation of the underlying neurocircuitry mechanism (Aceves-Piña and Quinn, 1979; Heisenberg et al., 1985; Tully et al., 1994; Vosshall and Stocker, 2007; Furukubo-Tokunaga and Hirth, 2012; Diegelmann et al., 2013). Previous works showed that associative olfactory memory could be induced in larvae either by the two-odor reciprocal protocol (Scherer et al., 2003; Hendel et al., 2005; Michels et al., 2005; Selcho et al., 2009, 2014; Gerber et al., 2010; Diegelmann et al., 2013) or by the single-odor nonreciprocal protocol (Honjo and Furukubo-Tokunaga, 2005, 2009; Khurana et al., 2009; Kleber et al., 2016). While many studies have utilized the two-odor reciprocal protocol, it involves several intricate handling steps (Hendel et al., 2005; Michels et al., 2005; Selcho et al., 2009, 2014; Pauls et al., 2010; Diegelmann et al., 2013). On the other hand, although care need 
to be taken for choosing controls, the single-odor protocol consists of fewer training cycles (Honjo and FurukuboTokunaga, 2005, 2009; Khurana et al., 2009; Pauls et al., 2010).

In this protocol, based on the singe-odor paradigm (Honjo and Furukubo-Tokunaga, 2005, 2009), we applied the optogenetic and thermogenetic techniques to induce associative olfactory memory in larvae without behavioral conditioning. Previous works showed that synaptic output from octopaminergic (OA) neurons is necessary and sufficient for appetitive memory formation in larvae (Schroll et al., 2006; Honjo and Furukubo-Tokunaga, 2009; Selcho et al., 2014). Signals from OA neurons are integrated with the olfactory information pathway at two prominent brain structures, the antennal lobes and mushroom bodies, to establish appetitive associative memory (Heisenberg, 2003; Davis, 2011; FurukuboTokunaga and Hirth, 2012; Guven-Ozkan and Davis, 2014). It was also shown that light-induced stimulation of olfactory receptor neurons (ORNs) induces olfactory response behaviors in living larvae (Bellmann et al., 2010; Störtkuhl and Fiala, 2011).

Here, we describe step-by-step procedures of our protocol, which consists of (1) substitution of gustatory reward by thermogenetic activation of OA neurons with the Drosophila Transient receptor potential A1 (dTrpA1) channel (Hamada et al., 2008) under the control of the tyrosine decarboxylase 2 $(T d c 2)$ promoter, and (2) substitution of odor stimuli by optical activation of a specific class ORNs with Channelrhodopsin 2 (ChR2; Nagel et al., 2005), which is expressed under the control of a specific olfactory receptor (Or) promoter (Honda et al., 2014; Figures 1, 2A). After a brief paired conditioning with blue light and warm temperature, dual transgenic larvae (Or-ChR2; Tdc2$d \operatorname{TrpA1)}$ exhibit significant suppression of negative phototaxis toward blue light. The unpaired presentation of blue light and heat causes no alteration in phototaxis behaviors, suggesting that associative memory is induced only by a concomitant activation of the converging set of the endogenous neurons involved in memory formation.

While conventional behavioral protocols involve multiple steps of larval preparation and conditioning by hands, this artificial activation protocol consists of minimum operational steps requiring only $60 \mathrm{~s}$ for memory induction. Despite this very short conditioning, olfactory memory produced by this protocol is robust and as stable as the memory produced with the combination of a natural odorant and reward. We also describe a light-based memory assay using a blue/red LED plate, which provides us with a sensitive alternative to the conventional olfactory test on an agar plate.

\section{MATERIALS AND EQUIPMENT}

All the materials and equipment used in this protocol are listed in Table 1.

\section{Stepwise Procedures}

The workflow of this protocol is shown in Figure 1.

\section{Transgenic flies and lighting apparatus}

1.1. Description of the transgenic flies used in this protocol: In order to express ChR2 in a specific set of ORNs, we cloned the coding region of ChR2 under the specific promoter sequence of specific Or genes (Wang et al., 2003; Larsson et al., 2004; Fishilevich et al., 2005; Kreher et al., 2005, 2008; Or-ChR2 in Figure 2B). In order to express dTrpA1 in reward neurons (Hamada et al., 2008), we cloned the $\mathrm{dTrpA} 1$ coding sequence under the promoter of the $T d c 2$ gene (Cole et al., 2005; Tdc2-dTrpA1 in Figure 2B). See the construct design described in the Supplemental Figure 1.

Note: The Tdc2 promoter is known to drive specific expression in $\mathrm{OA}$ neurons in the larval brain (Honjo and Furukubo-Tokunaga, 2009; Selcho et al., 2012, 2014; Zhang et al., 2013). We have deposited these stocks at the Bloomington Stock Center (Indiana, USA). The standard stock used in this protocol is $\mathrm{w}$ (CS10), which was outcrossed with Canton S. All stocks were outcrossed to $\mathrm{w}(\mathrm{CS} 10)$ at least five times before experiments.

Optional: Setting up genetic crossing with mutant flies. The transgenic larvae are homozygous for both Or-ChR2 (2nd chromosome) and Tdc2-dTrpA1 (third chromosome), which can be combined with either gain-of-function or lossof function background by standard genetic crossing. Binary expression systems such as GAL4-UAS can be used to drive an overexpression or RNAi constructs in conjunction with this protocol (see Discussion).

1.2. Fly stock maintenance: Raise the flies with a standard corn meal food $(5.5 \mathrm{~g} / \mathrm{L}$ agar, $40 \mathrm{~g} / \mathrm{L}$ yeast extract, 90 g/L cornmeal, $100 \mathrm{~g} / \mathrm{L}$ glucose, and $0.7 \mathrm{~g} / \mathrm{L}$ n-butyl-phydroxybenzoate). Maintain fly stocks in an incubator set to $25^{\circ} \mathrm{C}, 75 \%$ relative humidity and under dark or red $\operatorname{dim}$ light.

1.3. Lighting equipment: Set up a swan-neck lighting apparatus with light-emitting diodes (LEDs) (3 Watt each) on dual flexible arms (Figures 2C, 3G).

1.4. Blue/red LED quadrants plate: Prepare diametrically opposed quadrants, each illuminated with LEDs of 468 $\mathrm{nm}$ LED $(2600 \mathrm{mcd})$ in the blue quadrants covered by a $490 \mathrm{~nm}$ short-path filter, and with $625 \mathrm{~nm}$ LED (2600 $\mathrm{mcd}$ ) in the red quadrants covered with $580 \mathrm{~nm}$ long-path filter (Figures 2, 3). To ensure homogeneous illumination, diffusion filter of $50 \%$ transmission is placed on the LED units (Figure 3). The light intensity on the surface of the agar plate is approximately 2900 lux as measured with a digital illuminometer.

1.5. Agar plates: Make agar plates by dissolving $7.5 \mathrm{~g}$ agar powder in $300 \mathrm{~mL}$ deionized water (2.5\% agar), heat up with a microwave and then pour $20 \mathrm{~mL}$ to each $90 \mathrm{~mm}$ petri dish. The agar plates can be kept at $4^{\circ} \mathrm{C}$ till the next day.

\section{Preparation of early third instar larvae}

2.1. Prepare $100 \mathrm{mM}$ all-trans-retinal stock. Dissolve all-transretinal $(100 \mathrm{mg})$ in $100 \%$ ethanol $(3.52 \mathrm{~mL})$. Aliquot the stock solution at $1 \mathrm{~mL}$ in $1.5 \mathrm{~mL}$ test tubes wrapped with aluminum foil, and store in a lightproof box at $-20^{\circ} \mathrm{C}$.

2.2. Make all-trans-retinal food. Melt corn meal food $(10 \mathrm{~mL}$ corn meal food per vial) in a microwave oven, and add 100 $\mu \mathrm{L}$ all-trans-retinal (100 mM) per vial (final $1 \mathrm{mM})$. Mix well to ensure homogeneity. 


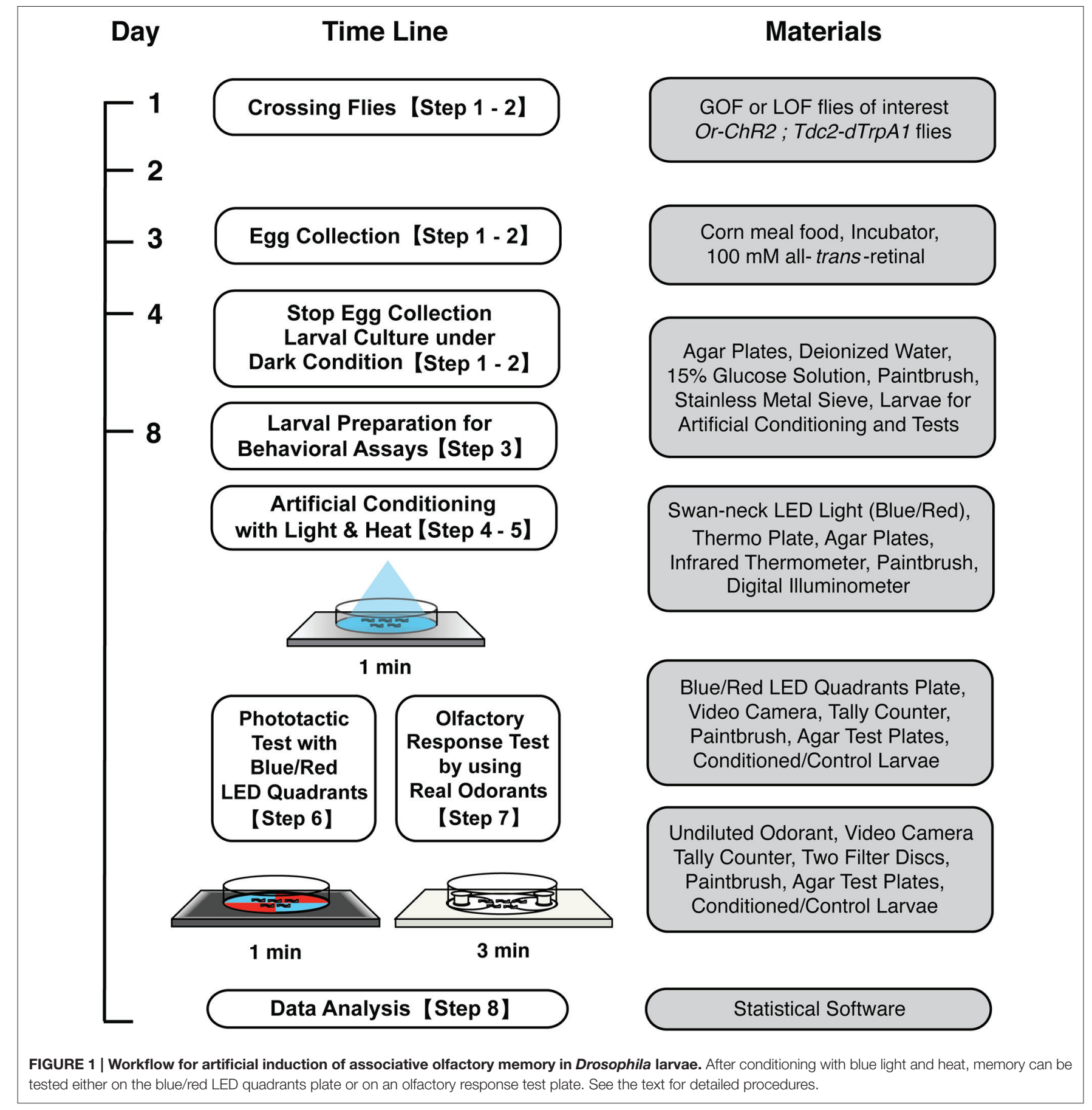

2.3. Collect eggs for $4 \mathrm{~h}$ at $25^{\circ} \mathrm{C}$ under dark from adult flies of 3-10 days old. Do not include more than 50 flies in the vial to avoid overcrowding of emerging larvae.

2.4. Raise larvae in all-trans-retinal containing food at $25^{\circ} \mathrm{C}$ under dark for $72 \mathrm{~h}$ after the end of the egg collection (early third instar larvae).

Note: We suggest using early third instar larvae (72-76 h after egg laying) because of easier handling compared to the earlier stages. Late third instar larvae could be used but care should be taken for staging to avoid the ecdysone effect.

3. Harvesting larvae for conditioning

3.1. All experiments on step 3-7 should be conducted at $25^{\circ} \mathrm{C}$ room temperature.

3.2. Preset the thermo plate at $36^{\circ} \mathrm{C}$. Put an agar test plate on the thermo plate and check the surface temperature of agar to be $28^{\circ} \mathrm{C}$ with an infrared thermometer. Adjust the hot plate 
A

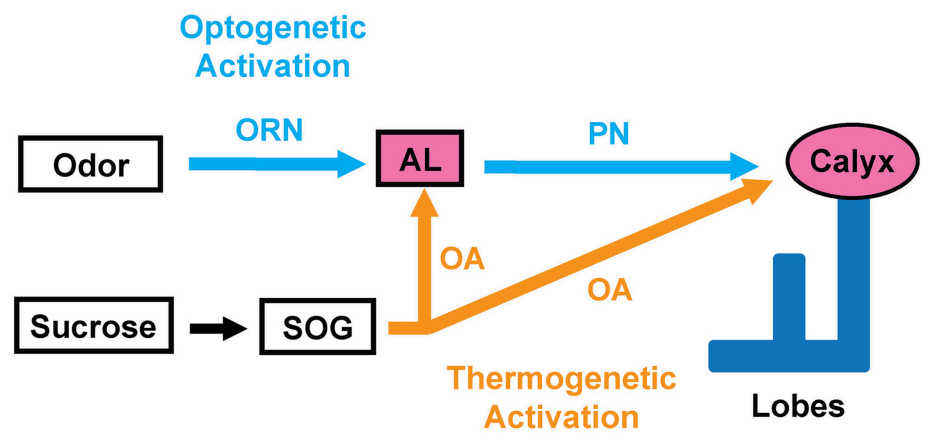

B

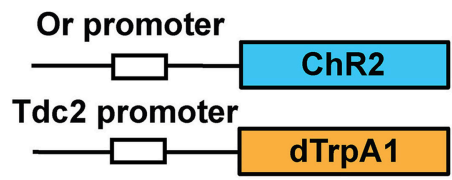

C

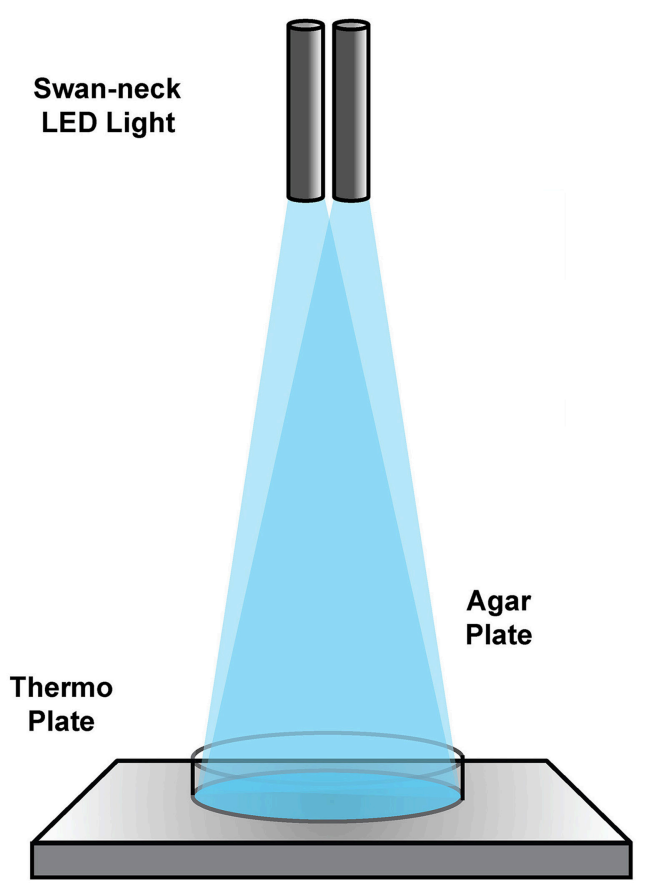

D
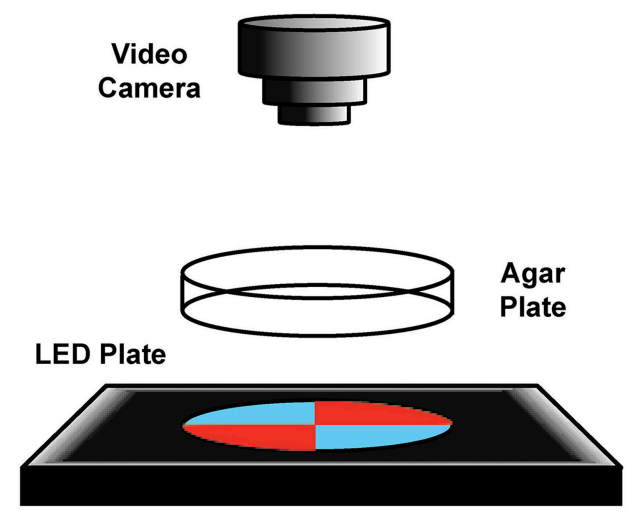

E

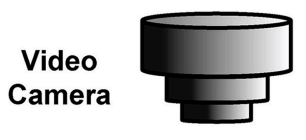

Filter

Disk

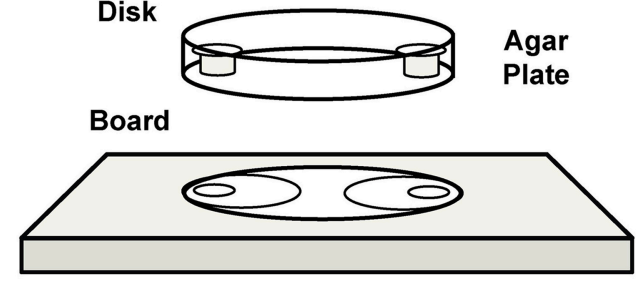

FIGURE 2 | Transgenic constructs and LED apparatus. (A) Schematic diagram for artificial induction of associative olfactory memory in the fruit fly larvae. This method consists of (1) substitution of reward signals with dTrpA1-mediated thermogenetic activation of OA neurons and (2) substitution of the odor signals with ChR2-mediated optical activation of a specific class of ORNs. (B) Structures of the Or-ChR2 and Tdc2-dTrpA1 constructs. Or-ChR2 construct; the coding sequence of ChR2 is directly placed under the transcriptional promoter sequence of an Or gene, which is expressed in a specific set of the larval ORNs. Tdc2-dTrpA1 construct; the coding sequence of $d T r p A 1$ is fused with the transcriptional promoter sequence of the Tdc2 gene, which is expressed in the larval OA neurons. (C) Setup for artificial conditioning with blue light and heat. Swan-neck LED light and thermo plate. (D) blue/red LED quadrants plate. (E) Olfactory response test plate.

setting as required. Exact setting of the thermo plate may depend on the heating apparatus. Note also that it may take several minutes to have the equilibrium of the agar surface temperature.
3.3. Harvest early third instar larvae by pouring $15 \%$ glucose solution into vials, and gently disturb the food surface with a spatula to release larvae into the solution. 
TABLE 1 | Materials and equipments.

\begin{tabular}{|c|c|c|}
\hline Name of material/equipment & Company & Catalog/model number \\
\hline All-trans-retinal & Sigma-Aldrich, MO, USA & R2500 \\
\hline Agar powder & Nacalai tesque, Tokyo, Japan & $01028-85$ \\
\hline Glucose & Sigma-Aldrich, MO, USA & G7021 \\
\hline $100 \%$ Ethanol & Nacalai tesque, Tokyo, Japan & $14713-95$ \\
\hline Acetophenone & Nacalai tesque, Tokyo, Japan & $00412-52$ \\
\hline Ethyl acetate & Nacalai tesque, Tokyo, Japan & $14747-65$ \\
\hline Digital video camera & SONY, Tokyo, Japan & HDR-CX520 \\
\hline Digital illuminometer & AS ONE, Tokyo, Japan & AR-813A \\
\hline Filter paper, $\varnothing 70 \mathrm{~mm}$ (Cut and use in $\varnothing 10 \mathrm{~mm}$ ) & ADVANTEC, Tokyo, Japan & 00021070 \\
\hline Petri dish, $90 \times 15 \mathrm{~mm}$ & IWAKI, Tokyo, Japan & $36-3412$ \\
\hline Thermo Plate & TOKAI HIT, Tokyo, Japan & MATS-SPE \\
\hline Twin LED light (Blue/Red) & RelyOn, Tokyo, Japan & Order-made \\
\hline Infrared radiometer & Testo AG, Baden-Württemberg, Germany & 830-T1 \\
\hline $468 \mathrm{~nm}$ Blue LEDs (2600 mcd) with $490 \mathrm{~nm}$ short-path filter of $50 \%$ transmission & RelyOn, Tokyo, Japan & Order-made \\
\hline $625 \mathrm{~nm}$ Red LEDs (2600 mcd) with $580 \mathrm{~nm}$ long- path filter of $50 \%$ transmission & RelyOn, Tokyo, Japan & Order-made \\
\hline GraphPad prism 6 & GraphPad Software, CA, USA & MDF-GP6ECO \\
\hline
\end{tabular}

3.4. Collect the larvae on a stainless metal sieve (diameter $10 \mathrm{~cm}$, aperture, $500 \mu \mathrm{m}$ ) by decantation. Remove food debris with forceps.

3.5. Transfer the larvae to $100 \mathrm{~mL}$ beaker by running distilled water from a washing bottle and using a paintbrush. Rinse the larvae three times gently with distilled water to remove food debris and glucose.

3.6. Transfer the larvae on a fine metal mesh $(10 \times 10 \mathrm{~cm}$, aperture $\sim 100 \mu \mathrm{m}$ ) with a paintbrush. Briefly touch paper towel on the other side of the mesh to remove the excess fluid.

3.7. Transfer the larvae to an agar plate $\left(21^{\circ} \mathrm{C}\right)$ with a paintbrush. The prepared larvae can be kept on the agar up to $10 \mathrm{~min}$ before conditioning.

\section{Conditioning with blue light and heat}

4.1. Place a fresh agar plate on the thermo plate pre-adjusted in step 3.2. Ensure the surface temperature of agar is $28^{\circ} \mathrm{C}$ with an infrared thermometer.

4.2. Adjust the illumination of the swan-neck LED light (blue) to cover the entire surface of the agar plate (Figure 3G).

Note: Set the light intensity on the agar surface at 8000 lux as measured with a digital illuminometer. Subsequent steps (4.3-6.2) should be conducted in the dark with a safe red light ( $\sim 1$ lux) for photographic work.

4.3. Gently place $\sim 50$ larvae on the pre-warmed agar plate with a paintbrush (Figure 2C).

4.4. Illuminate the animals for $60 \mathrm{~s}$ (continuous) with the swanneck LED light (blue; Figures 2C, 3G).

Note: Either longer ( $\geq 90 \mathrm{~s}$ ) or shorter ( $\leq 45 \mathrm{~s}$ ) illumination results in poor conditioning (Honda et al., 2014).

\section{Dissociation and light/heat control tests}

5.1. Dissociation tests: In order to verify associative conditioning, perform the following set of experiments, in which animals are exposed to both heat $\left(28^{\circ} \mathrm{C}\right)$ and blue light but either unpaired or paired way (See the schematic diagrams in Figures 4A, 5A).
(1) Place an agar plate on the thermo-plate and adjust the temperature of the agar surface either at $28^{\circ} \mathrm{C}$ (for unpaired conditioning) or at $21^{\circ} \mathrm{C}$ (for paired conditioning). Gently place $\sim 50$ larvae on the agar plate with a paintbrush. Illuminate the animals for $60 \mathrm{~s}$ with red light.

(2) After the first conditioning, transfer the larvae to an agar plate pre-adjusted at $21^{\circ} \mathrm{C}$. Keep the larvae for $3 \mathrm{~min}$ without light simulation.

(3) After the interval, transfer the larvae to another agar plate pre-adjusted either at $21^{\circ} \mathrm{C}$ (for unpaired conditioning) or at $28^{\circ} \mathrm{C}$ (for paired conditioning), and illuminate them for $60 \mathrm{~s}$ with blue light.

(4) Proceed to Step 6 or 7 for memory test at $21^{\circ} \mathrm{C}$.

5.2. Light/heat control tests:

To separately examine the effects of light and heat exposures, perform the following sets of conditioning (Supplemental Figure 2A) based on the procedures described in Step 4 but with different light/heat parameters. These experiments involve controls of basic larval handlings, which would help troubleshooting when the dissociation tests fail to work.

$$
\begin{aligned}
& \text { Blue light at } 21^{\circ} \mathrm{C} \\
& \text { Red light at } 28^{\circ} \mathrm{C} \\
& \text { Red light at } 21^{\circ} \mathrm{C}
\end{aligned}
$$

\section{Larval phototaxis test with blue/red LED quadrants}

6.1. After conditioning, transfer the larvae with a paintbrush to the center of a fresh agar plate on the blue/red LED quadrants apparatus (Phototaxis test plate; Figures 2D, 3E), and leave the animals to choose the blue/red quadrants.

6.2. After $1 \mathrm{~min}$, count the number of larvae in the blue and red quadrants. Exclude the larvae on the border of blue and red quadrants and the larvae crawling up the peripheral wall of the plastic plate from counting as they may be out of proper lighting.

6.3. Calculate the Response Index (RI) based on the following equation. 


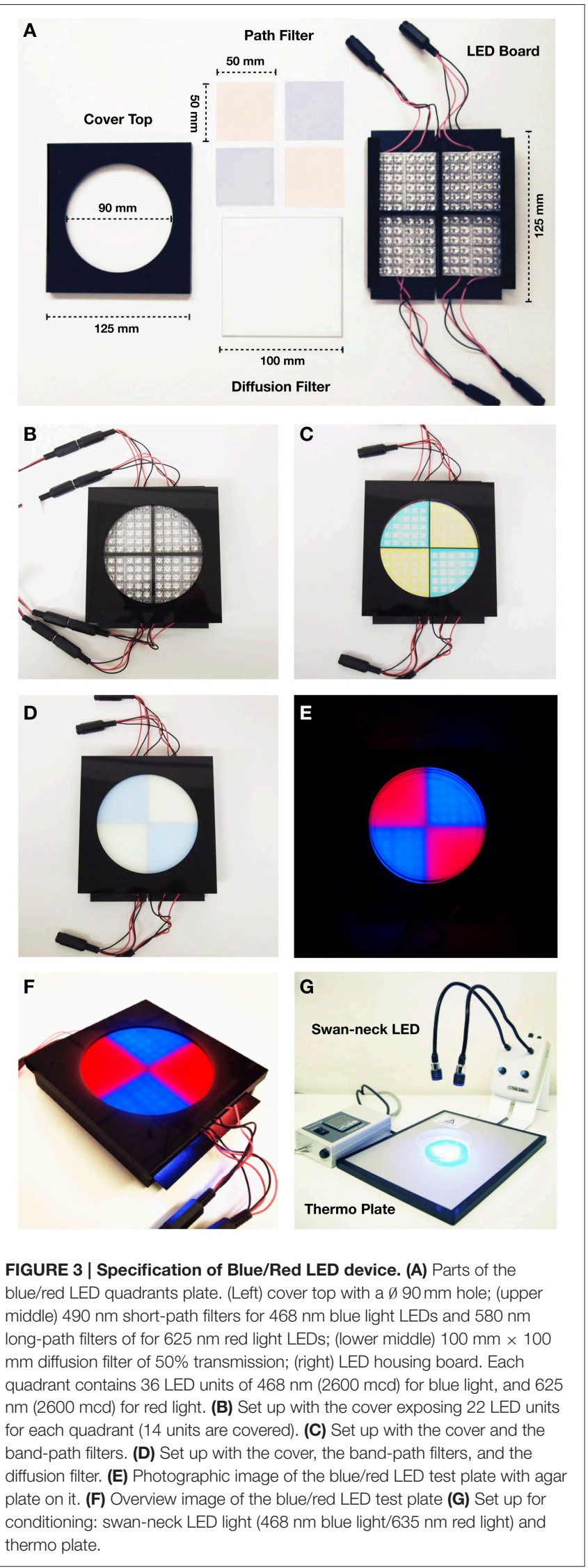

$\mathrm{RI}=\left(\mathrm{N}^{\text {Blue }}-\mathrm{N}^{\text {Red }}\right) /\left(\mathrm{N}^{\text {Blue }}+\mathrm{N}^{\text {Red }}\right)$

$\mathrm{N}^{\text {Blue }}$ : The number of larvae in the blue quadrants

$\mathrm{N}^{\text {Red }}$ : The number of larvae in the red quadrants

\section{Olfactory Preference test}

7.1. Perform olfactory tests to verify odor-specificity and to exclude the possibility of general desensitization toward blue light (Figure 5A and Supplemental Figure 3A). The olfactory response test described here is based on the agar plate assay described previously (Aceves-Piña and Quinn, 1979; Rodrigues, 1980; Monte et al., 1989), and can be conducted under normal light condition ( $\sim 400$ lux).

Note: Some of the odorants are potentially harmful and flammable. They should be dispensed to a small quantity $(10 \mu \mathrm{L})$ in the fume hood before experiments.

7.2. Prepare a fresh agar plate $(2.5 \%, 90 \mathrm{~mm})$. Place a lid of a 1.5 $\mathrm{mL}$ microcentrifuge tube on both sides, $5 \mathrm{~mm}$ away from the outer edge (Figure 2E and Supplemental Figure 3A), and put paper filter discs ( $10 \mathrm{~mm}$ diameter) on them. Avoid touching the lid and filter discs with bare hands.

7.3. After conditioning with blue light and heat (step 4), transfer $\sim 50$ animals to the center of the test plate with a paintbrush (Figure 5A and Supplemental Figure 3A). Spread tangled animals gently with a paintbrush. Spot $2.5 \mu \mathrm{L}$ undiluted odorant on the test disc and none on the control disc. Close the lid immediately.

7.4. After $3 \mathrm{~min}$, count the number of larvae inside of the semicircular areas $(3 \mathrm{~cm}$ in radius from each filter disc).

7.5. Calculate the RI based on the following equation.

$$
\begin{aligned}
& \mathrm{RI}=\left(\mathrm{N}^{\text {Odor }}-\mathrm{N}^{\text {Control }}\right) /\left(\mathrm{N}^{\text {Odor }}+\mathrm{N}^{\text {Control }}\right) \\
& \mathrm{N}^{\text {Odor }} \text { : The number of larvae in the odor area } \\
& \mathrm{N}^{\text {Control }} \text { : The number of larvae in the control area }
\end{aligned}
$$

\section{Data analysis}

8.1. Calculation of memory performance scores: To analyze the extent of behavioral changes, calculate the memory performance score based on the following equation (Kleber et al., 2016; Figures 4C, 5C).

$$
\text { Performance Index }=\left(\mathrm{RI}^{\text {Paired }}-\mathrm{RI}^{\text {Unpaired }}\right) / 2
$$

8.2. Statistics: Perform power calculation to determine the exact sample size (number of trials) necessary for the sufficient effect size and statistical power. This would require performing larval memory tests at least 10 times.

8.3. Examination of data distribution: Since the obtained Response Index (RI) is not always normally distributed, normality tests such as the D'Agostino-Pearson (Omnibus K2) test should be performed against the obtained data.

If the data set shows a Gaussian distribution $(P \geq 0.05$ in normality tests), perform parametric test such as Student's $t$-test (for pair-wise comparisons) and ANOVA followed by Tukey's post-hoc test (for multiple comparisons). If the 


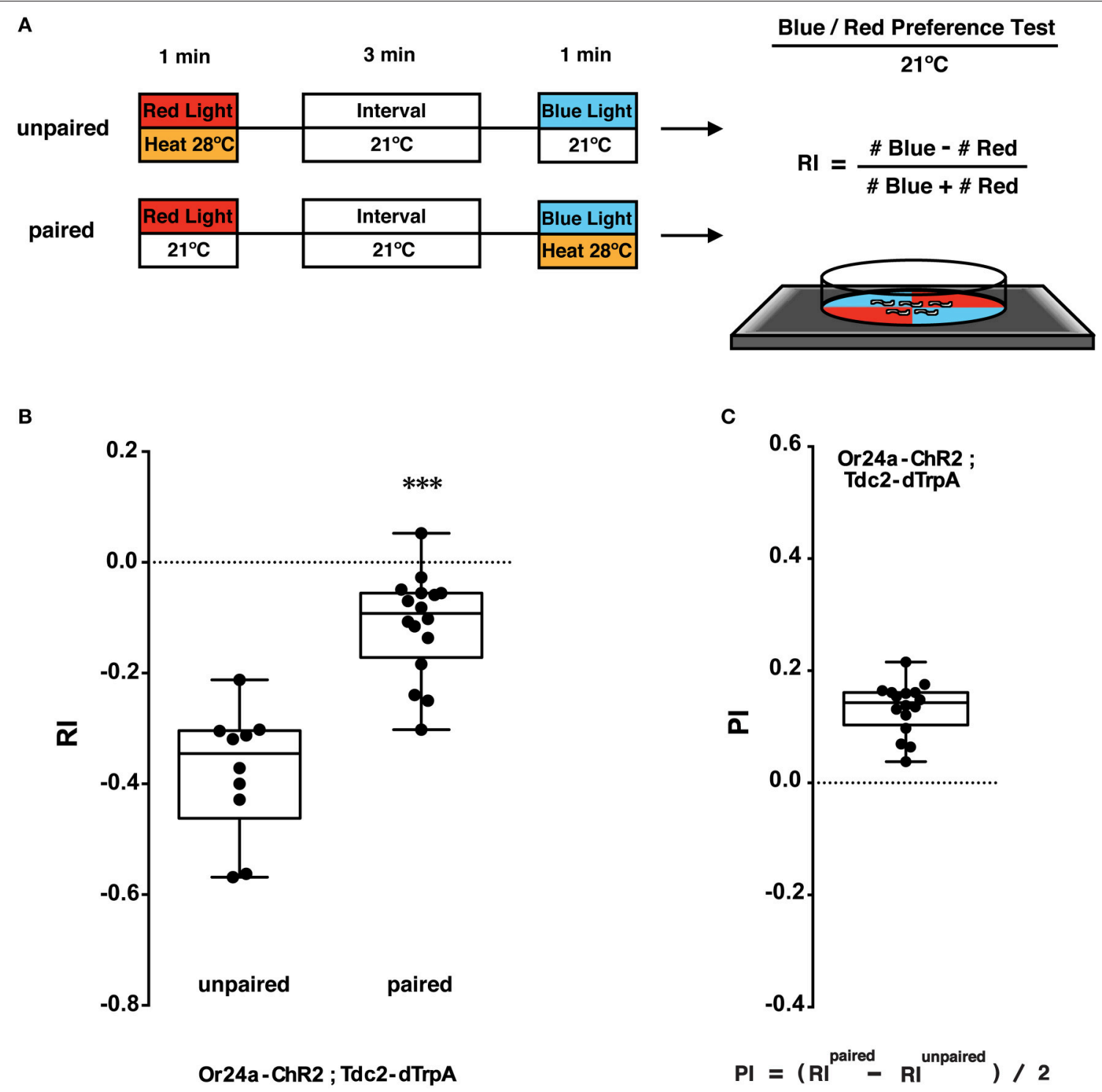

FIGURE 4 | Phototactic tests of larval memory formed with paired presentation of blue light and heat. (A) Larval conditionings and phototaxis test. Transgenic larvae carrying both Or-ChR2 and Tdc2-dTrpA1 were conditioned with heat $\left(28^{\circ} \mathrm{C}\right)$ and blue light in either unpaired or paired way. After the first conditioning with red light $(60 \mathrm{~s})$, larvae are transferred to an interval plate $\left(21^{\circ} \mathrm{C}\right)$, and then transferred to another plate to be illuminated with blue light for $60 \mathrm{~s}$. Heat stimulation was applied during either the first conditioning (unpaired) or the second conditioning (paired with blue light). Larval phototactic behavior was then tested at $21^{\circ} \mathrm{C}$ on the blue/red quadrants plate. (B) Phototactic responses of transgenic larvae (Or24a-ChR2; Tdc2-dTrpA1) with unpaired and paired conditioning. The paired but not the unpaired conditioning with blue light and heat $\left(28^{\circ} \mathrm{C}\right)$ caused significant suppression of negative phototaxis toward blue light. ${ }^{* \star *} p<0.001$ by Mann-Whitney $U$-test between unpaired and paired group. $n=10-16$ trials. (C) Memory performance scores (PI) of the paired conditioning group against the unpaired control. Box plots represent the median as the middle line, 25th and 75th percentile as box boundaries, as well as the minimum and the maximum as whiskers, respectively.

data set fails to show a Gaussian distribution, perform non-parametric tests such as Mann-Whitney U-test (for pair-wise comparisons) and Kruskal-Wallis test followed by Dunn's post-hoc test (for multiple comparisons).

\section{ANTICIPATED RESULTS}

\section{Memory Test with Blue/Red LED Quadrants}

The larvae were stimulated with blue light and heat $\left(28^{\circ} \mathrm{C}\right)$ in unpaired and paired ways (Figure 4A). Concomitant stimulation with blue light and heat caused significant suppression of negative phototaxis toward blue light as compared to unpaired exposure to both stimuli ( $p<0.001$, Figure 4B). A comparison between paired- and unpaired-trained groups revealed significant associative memory with the positive PI value (Figure 4C). Associative memory performance was assessed using the equation (RI $\left.{ }^{\text {Paired }}-\mathrm{RI}^{\text {Unpaired }}\right) / 2$ (Kleber et al., 2016). These results showed that associative memory was induced only by a concomitant activation of the converging set of the endogenous neurons involved in memory formation. 
A

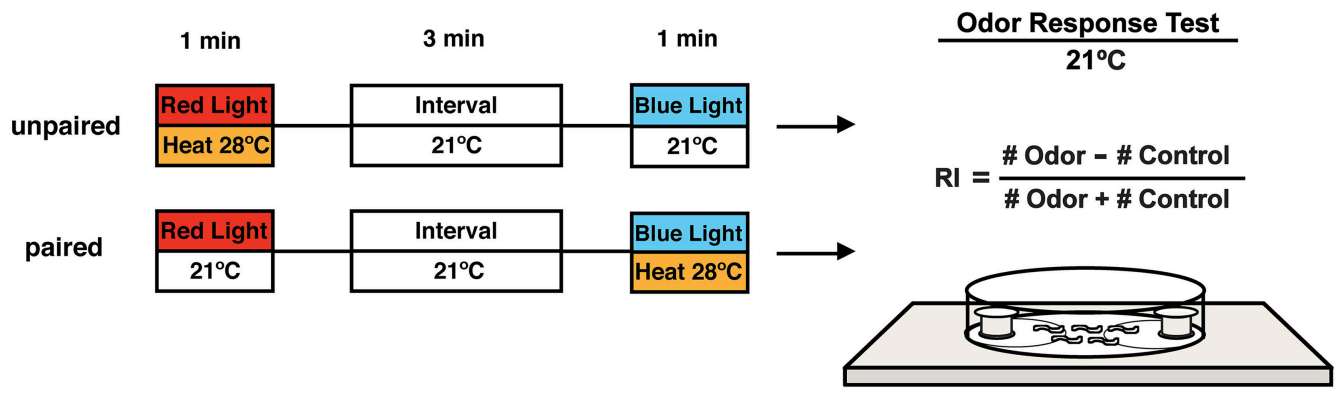

B

Or24a-ChR2 ; Tdc2-dTrpA

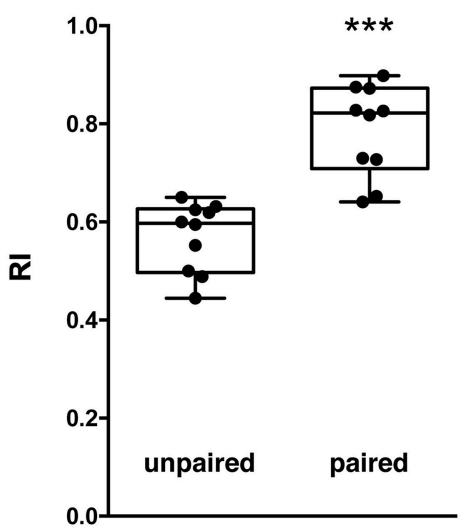

Acetophenone
Or42b-ChR2; Tdc2-dTrpA

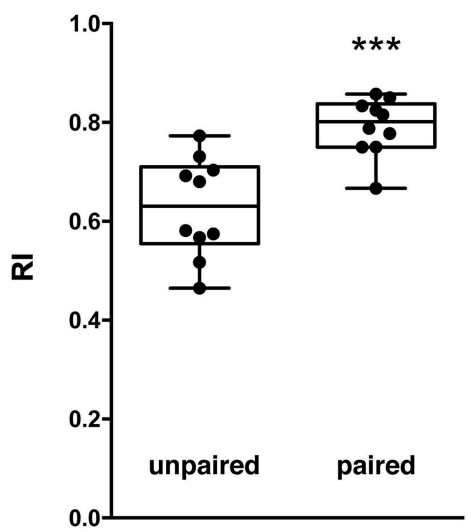

Ethyl Acetate

C
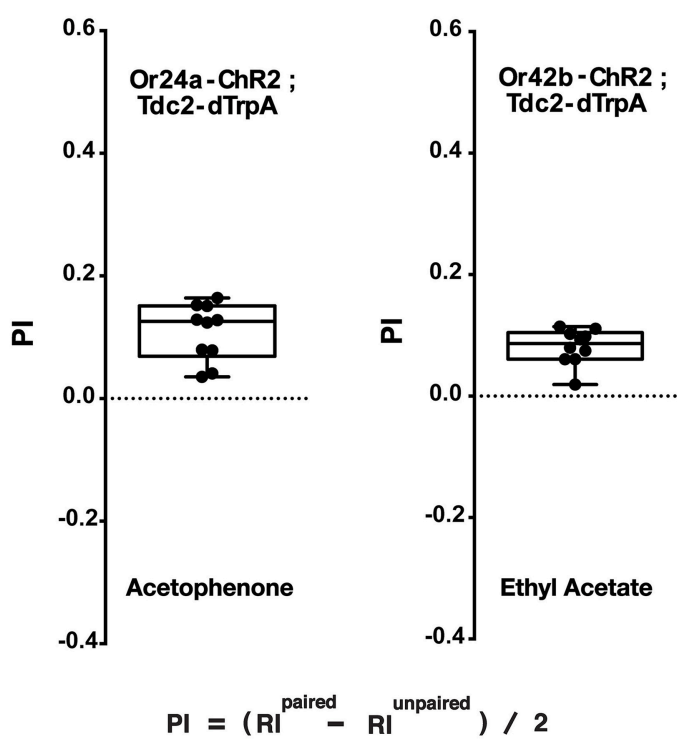

FIGURE 5 | Olfactory response tests of larval memory formed with paired presentation of blue light and heat. (A) Larval conditionings and olfactory test. Transgenic larvae carrying both Or-ChR2 and Tdc2-dTrpA1 were conditioned with heat $\left(28^{\circ} \mathrm{C}\right)$ and blue light in either unpaired or paired way as described in

Figure 4A. Larval olfactory response was then tested at $21^{\circ} \mathrm{C}$ on the olfactory test plate. (B) Olfactory responses of transgenic larvae (Or-ChR2; Tdc2-dTrpA1) with unpaired and paired conditioning. The paired but not the unpaired conditioning with blue light and heat $\left(28^{\circ} \mathrm{C}\right)$ caused significant increase in the olfactory response to the specific odorant determined by the Or-ChR2 type. ${ }^{* \star *} p<0.001$ by Mann-Whitney U-test between unpaired and paired group. $n=10$ trials. (C) Memory performance scores (PI) of the paired conditioning group against the unpaired control. Box plots represent the median as the middle line, 25th and 75th percentile as box boundaries, as well as the minimum and the maximum as whiskers, respectively. 
As light/heat controls, animals were stimulated for $60 \mathrm{~s}$ with either blue or red light in combination with 21 or $28^{\circ} \mathrm{C}$ (light/heat controls; Supplemental Figure 2A). The phototactic behavior of the dual transgenic larvae (Or-ChR2; $T d c 2-d \operatorname{Tr} p A 1)$ was not altered by red light at either temperature (Supplemental Figure 2B). By contrast, blue light stimulation at $28^{\circ} \mathrm{C}$ but not at $21^{\circ} \mathrm{C}$ caused significant suppression of negative phototaxis toward blue light ( $p<0.001$, Supplemental Figure 3B). No difference on RI was observed for the $w$ (CS10) control larvae by either conditioning (Supplemental Figure 2B).

\section{Memory Test with Specific Odorants}

The animals were stimulated with blue light and heat $\left(28^{\circ} \mathrm{C}\right)$ in unpaired and paired ways (Figure 5A). Only the paired stimulation with blue light and heat transgenic larvae (Or24aChR2; Tdc2-dTrpA1 and Or42b-ChR2; Tdc2-dTrpA1) resulted in significant increases in the olfactory response toward the specific odorant, acetophenone or ethyl acetate, respectively ( $p$ $<0.001$, Figures 5B,C). These results demonstrated that the artificial memory was specific to the odorant determined by the type of the ORNs stimulated with blue light.

Compared to the control ( $60 \mathrm{~s}$ blue light at $21^{\circ} \mathrm{C}$ ), associative conditioning $\left(60 \mathrm{~s}\right.$ blue light at $28^{\circ} \mathrm{C}$ ) of transgenic larvae resulted in significant increases in the olfactory response toward the odorant predicted by the Or-ChR2 type $(p<0.01$, Supplemental Figure 3B). Transgenic larvae carrying Or24a-ChR2 showed increased response toward acetophenone while transgenic larvae carrying Or42b-ChR2 showed increased response toward ethyl acetate (Supplemental Figure 3B).

Statistical calculations were performed using Prism 6 (GraphPad, San Diego, CA, USA). Further examples of experimental results can be found in Honda et al. (2014).

\section{DISCUSSION}

Various techniques have been published for olfactory associative learning in Drosophila larvae (Aceves-Piña and Quinn, 1979; Heisenberg et al., 1985; Tully et al., 1994; Scherer et al., 2003; Schwaerzel et al., 2003; Hendel et al., 2005; Honjo and FurukuboTokunaga, 2005; Michels et al., 2005; Vosshall and Stocker, 2007; Honjo and Furukubo-Tokunaga, 2009; Khurana et al., 2009; Selcho et al., 2009; Gerber et al., 2010; Furukubo-Tokunaga and Hirth, 2012; Diegelmann et al., 2013; Selcho et al., 2014; Kleber et al., 2016). It has been shown that $T \beta H$ mutant larvae exhibit abnormal locomotor behaviors (Saraswati et al., 2004; Selcho et al., 2012), and that ablation of the Tdc2 neurons in the ventral nervous system impairs larval locomotion (Selcho et al., 2012). However, in contrast to the locomotor deficits caused by chronic inactivation, acute activation of the $T d c 2$ neurons (60 s) during conditioning did not alter the larval phototactic and olfactory responses in our protocol unless it was paired with the activation of the Or neurons (Figures 4, 5). These results were also confirmed by the light/heat control data (Supplemental Figure 2B), which showed that $60 \mathrm{~s}$ heat stimulation under red light did not alter larval response, and consistent with the results by Schroll et al., which show that temporal light activation of the OA/tyramine neurons did not impair odor perception and locomotor activity (Schroll et al., 2006).

\section{Significance with Respect to Existing Methods}

Unlike the conventional behavioral protocols, our protocol selectively stimulates the target neurons for memory induction, and thus significantly reduces the background neuronal activities. It is also noteworthy that conventional animal training with natural odorants leads to activation of multiple ORN classes due to the redundancy in Or affinities to diverse odors (Kreher et al., 2005, 2008; Hallem et al., 2006), which generates complex olfactory codes in the memory centers. By contrast, stimulating only a single type ORNs, the protocol described in this work provides us with a highly simplified setup for the olfactory code, which would be of help for high-resolution analyses of neural circuits involved in memory dynamics in the fly brain.

In addition, since animals are conditioned remotely, this technique can be adapted for memory experiments under the microscope using dissected brains. In such cases, formation of associative memory could be identified with optical activity reporters, such as GCaMP (Broussard et al., 2014) or CaMPARI (Fosque et al., 2015), expressed in the memory centers in the brain. Furthermore, because the transgenic larvae used in this technique do not involve the GAL4-UAS system, it allows complementary usage of various binary expression systems including not only the GAL4-UAS but also other expression systems such as LexA-LexAop or QF-QUAS (del Valle Rodríguez et al., 2012) either to monitor the activities of other neurons or to manipulate additional sets of neurons.

Lilly and Carlson (1990) showed that Drosophila larvae are negatively phototactic and partition onto the dark quadrants on the phototactic assay plate. We found similar negative phototaxis on the blue-red quadrants, where naïve larvae avoid blue light. Despite this innate behavior, we found that the phototaxis assay on the blue/red LED quadrants is highly sensitive to detect the behavioral alterations after the artificial conditioning by this protocol.

While we have utilized the $T d c 2$ promoter to drive $\operatorname{dTrpA} 1$ in OA/tyramine neurons, recent studies have shown that different sets of dopaminergic neurons mediate positive and negative values in the adult flies (Aso et al., 2010, 2012; Burke et al., 2012; Liu et al., 2012). It has been shown that the D1-DA receptor dDA1 is required for both aversive and appetitive learning in larvae (Kim et al., 2007; Selcho et al., 2009). Whereas the THGAL4 driver, which includes the PPL1 but not the PAM cluster neurons, fails to mediates reward leaning in larvae (Honjo and Furukubo-Tokunaga, 2009), a recent work (Rohwedder et al., 2016) has shown that reward value is mediated by PAM-like dopamine neurons (pPAM) in the larval brain. Direct activation of larval PAM neurons would be an attractive choice in future experiments.

\section{Critical Steps and Troubleshooting}

One of the most critical steps is the preparation of larvae in good conditions. Overcrowded cultures should not be used since they are likely to produce poorly behaving animals (Step 2.3). For 
efficient ChR2 stimulation, all-trans-retinal (final $1 \mathrm{mM}$ ) needs to be supplemented in the larval culture food (Step 2.2) (see also Honjo et al. (2012). Details of larval handling are also described in Monte et al. (1989).

Heating at high temperature $\left(>30^{\circ} \mathrm{C}\right)$ is noxious for larvae (Tracey et al., 2003). Precise measurement of the plate temperature using an infrared thermometer is recommended (Step 3.2 and 4.1). On the other hand, negative control experiments for dTrpAl stimulation should be performed at $21^{\circ} \mathrm{C}$, which is low enough to silence the channel. Associative conditioning with blue light is best achieved with $\sim 1 \mathrm{~min}$ illumination (Step 4.4). Longer illumination may lead to poor memory scores (Honda et al., 2014).

For both conditioning and response tests (Step 4-7), it is important not to apply more than 50 larvae on the plate to avoid excess animal tangling, which alters subsequent larval behavioral responses. Gentle spreading of tangled larvae is of help.

To ensure the combined effect of light and heat, we suggest performing the dissociation test (Step 5.1, Figures 4, 5; Honjo and Furukubo-Tokunaga, 2005; Kleber et al., 2016). These experiments involve stimulation of the transgenic larvae with both heat and blue light but in unpaired and paired ways, and thus essential to verify the associative effect of the unconditioned and conditioned stimuli.

For technical controls and troubleshooting, we suggest performing the light/heat controls (Step 5.2, Supplemental Figure 2), which examine the individual effect of heat and blue light. It also provides a control of basic larval handling without heat and ChR2 stimulation (red light at $21^{\circ} \mathrm{C}$ ).

\section{Limitations of the Protocol}

This protocol is designed for Drosophila larvae carrying both the Or-ChR2 and Tdc2-dTrpA1 transgenes. The protocol could be applied to adult flies with some modifications as ORNs can

\section{REFERENCES}

Aceves-Piña, E. O., and Quinn, W. G. (1979). Learning in normal and mutant Drosophila larvae. Science 206, 93-96. doi: 10.1126/science.206.4414.93

Aso, Y., Herb, A., Ogueta, M., Siwanowicz, I., Templier, T., Friedrich, A. B., et al. (2012). Three dopamine pathways induce aversive odor memories with different stability. PLoS Genet. 8:1002768. doi: 10.1371/journal.pgen.1002768

Aso, Y., Siwanowicz, I., Bräcker, L., Ito, K., Kitamoto, T., and Tanimoto, H. (2010). Specific dopaminergic neurons for the formation of labile aversive memory. Curr. Biol. 20, 1445-1451. doi: 10.1016/j.cub.2010.06.048

Bellmann, D., Richardt, A., Freyberger, R., Nuwal, N., Schwärzel, M., Fiala, A., et al. (2010). Optogenetically induced olfactory stimulation in Drosophila larvae reveals the neuronal basis of odor-aversion behavior. Front. Behav. Neurosci. 4:27. doi: 10.3389/fnbeh.2010.00027

Broussard, G. J., Liang, R., and Tian, L. (2014). Monitoring activity in neural circuits with genetically encoded indicators. Front. Mol. Neurosci. 7:97. doi: 10.3389/fnmol.2014.00097

Burke, C. J., Huetteroth, W., Owald, D., Perisse, E., Krashes, M. J., Das, G., et al. (2012). Layered reward signalling through octopamine and dopamine in Drosophila. Nature 492, 433-437. doi: 10.1038/nature11614

Cole, S. H., Carney, G. E., McClung, C. A., Willard, S. S., Taylor, B. J., and Hirsh, J. (2005). Two functional but noncomplementing Drosophila tyrosine decarboxylase genes: distinct roles for neural tyramine and octopamine in female fertility. J. Biol. Chem. 280, 14948-14955. doi: 10.1074/jbc.M414197200 be activated by illuminating the antennae, except for the Or24a ORN, which is specific for larvae (Fishilevich et al., 2005; Kreher et al., 2005, 2008). Alternatively, red-shifted variants of ChR (Lin et al., 2013a,b; Inagaki et al., 2014) could be used to generate Or-ChR ${ }^{\text {red }}$ flies that can be activated by optogenetic tools. The fly stocks used in this protocol are made available from the Bloomington Stock Center.

\section{AUTHOR CONTRIBUTIONS}

KF designed the project. TH and CL performed the experiments and optimized the protocol. $\mathrm{KH}$ constructed parts of the plasmid and examined the protocol. $\mathrm{TH}, \mathrm{KH}$, and KF wrote the manuscript. All authors reviewed the manuscript.

\section{ACKNOWLEDGMENTS}

We thank Dr. M. Yoshida-Kashikawa for her help in transgenic animal construction, Drs. K. Deisseroth, P. Garrity, and F. N. Hamada for plasmids, and Bloomington Stock Center for fly stocks, and L. Irving for reading the manuscript. TH was supported by Research Fellowship for Young Scientists (DC1), the Japan Society for the Promotion of Science (JSPS KAKENHI Grant Number 15J06369). This work was supported by Grantsin-Aid for Scientific Research, Ministry of Education, Culture, Sports, Science and Technology, Japan, to KF (MEXT KAKENHI Grant Number 24570229).

\section{SUPPLEMENTARY MATERIAL}

The Supplementary Material for this article can be found online at: http://journal.frontiersin.org/article/10.3389/fnbeh. 2016.00137

Davis, R. L. (2011). Traces of Drosophila memory. Neuron 70, 8-19. doi: 10.1016/j.neuron.2011.03.012

del Valle Rodríguez, A., Didiano, D., and Desplan, C. (2012). Power tools for gene expression and clonal analysis in Drosophila. Nat. Methods 9, 47-55. doi: $10.1038 /$ nmeth. 1800

Diegelmann, S., Klagges, B., Michels, B., Schleyer, M., and Gerber, B. (2013). Maggot learning and synapsin function. J. Exp. Biol. 216, 939-951. doi: $10.1242 /$ jeb. 076208

Fishilevich, E., Domingos, A. I., Asahina, K., Naef, F., Vosshall, L. B., and Louis, M. (2005). Chemotaxis behavior mediated by single larval olfactory neurons in Drosophila. Curr. Biol. 15, 2086-2096. doi: 10.1016/j.cub.2005. 11.016

Fosque, B. F., Sun, Y., Dana, H., Yang, C. T., Ohyama, T., Tadross, M. R., et al. (2015). Labeling of active neural circuits in vivo with designed calcium integrators. Science 347, 755-760. doi: 10.1126/science.1260922

Furukubo-Tokunaga, K., and Hirth, F. (2012). "Memory circuits in Drosophila," in Memory Mechanisms in Health and Disease, ed P. Giese (Singapore: World Scientific), 269-306.

Gerber, B., Biernacki, R., and Thum, J. (2010). "Odour-taste learning in larval Drosophila," in Drosophila Neurobiology, ed S. Waddell (New York, NY: Cold Spring Harbor Laboratory Press), 443-455.

Guven-Ozkan, T., and Davis, R. L. (2014). Functional neuroanatomy of Drosophila olfactory memory formation. Learn. Mem. 21, 519-526. doi: $10.1101 / \mathrm{lm} .034363 .114$ 
Hallem, E. A., Dahanukar, A., and Carlson, J. R. (2006). Insect odor and taste receptors. Annu. Rev. Entomol. 51, 113-135. doi: 10.1146/annurev.ento.51.051705.113646

Hamada, F. N., Rosenzweig, M., Kang, K., Pulver, S. R., Ghezzi, A., Jegla, T. J., et al. (2008). An internal thermal sensor controlling temperature preference in Drosophila. Nature 454, 217-220. doi: 10.1038/nature07001

Heisenberg, M. (2003). Mushroom body memoir: from maps to models. Nat. Rev. Neurosci. 4, 266-275. doi: 10.1038/nrn1074

Heisenberg, M., Borst, A., Wagner, S., and Byers, D. (1985). Drosophila mushroom body mutants are deficient in olfactory learning. J. Neurogenet. 2, 1-30. doi: 10.3109/01677068509100140

Hendel, T., Michels, B., Neuser, K., Schipanski, A., Kaun, K., Sokolowski, M. B., et al. (2005). The carrot, not the stick: appetitive rather than aversive gustatory stimuli support associative olfactory learning in individually assayed Drosophila larvae. J. Comp. Physiol. A Neuroethol. Sens. Neural Behav. Physiol. 191, 265-279. doi: 10.1007/s00359-004-0574-8

Honda, T., Lee, C. Y., Yoshida-Kasikawa, M., Honjo, K., and Furukubo-Tokunaga, K. (2014). Induction of associative olfactory memory by targeted activation of single olfactory neurons in Drosophila larvae. Sci. Rep. 4:4798. doi: 10.1038/srep04798

Honjo, K., and Furukubo-Tokunaga, K. (2005). Induction of cAMP response element-binding protein-dependent medium-term memory by appetitive gustatory reinforcement in Drosophila larvae. J. Neurosci. 25, 7905-7913. doi: 10.1523/JNEUROSCI.2135-05.2005

Honjo, K., and Furukubo-Tokunaga, K. (2009). Distinctive neuronal networks and biochemical pathways for appetitive and aversive memory in Drosophila larvae. J. Neurosci. 29, 852-862. doi: 10.1523/JNEUROSCI.1315-08.2009

Honjo, K., Hwang, R. Y., and Tracey, W. D. Jr. (2012). Optogenetic manipulation of neural circuits and behavior in Drosophila larvae. Nat. Protoc. 7, 1470-1478. doi: 10.1038/nprot.2012.079

Inagaki, H. K., Jung, Y., Hoopfer, E. D., Wong, A. M., Mishra, N., Lin, J. Y., et al. (2014). Optogenetic control of Drosophila using a red-shifted channelrhodopsin reveals experience-dependent influences on courtship. Nat. Methods 11, 325-332. doi: 10.1038/nmeth.2765

Khurana, S., Abu Baker, M. B., and Siddiqi, O. (2009). Odour avoidance learning in the larva of Drosophila melanogaster. J. Biosci. 34, 621-631. doi: 10.1007/s12038-009-0080-9

Kim, Y. C., Lee, H. G., and Han, K. A. (2007). D1 dopamine receptor dDA1 is required in the mushroom body neurons for aversive and appetitive learning in Drosophila. J. Neurosci. 27, 7640-7647. doi: 10.1523/JNEUROSCI.1167-07.2007

Kleber, J., Chen, Y. C., Michels, B., Saumweber, T., Schleyer, M., Kähne, T., et al. (2016). Synapsin is required to "boost" memory strength for highly salient events. Learn. Mem. 23, 9-20. doi: 10.1101/lm.039685.115

Kreher, S. A., Kwon, J. Y., and Carlson, J. R. (2005). The molecular basis of odor coding in the Drosophila larva. Neuron 46, 445-456. doi: 10.1016/j.neuron.2005.04.007

Kreher, S. A., Mathew, D., Kim, J., and Carlson, J. R. (2008). Translation of sensory input into behavioral output via an olfactory system. Neuron 59, 110-124. doi: 10.1016/j.neuron.2008.06.010

Larsson, M. C., Domingos, A. I., Jones, W. D., Chiappe, M. E., Amrein, H., and Vosshall, L. B. (2004). Or83b encodes a broadly expressed odorant receptor essential for Drosophila olfaction. Neuron 43, 703-714. doi: 10.1016/j.neuron.2004.08.019

Lilly, M., and Carlson, J. (1990). Smellblind: a gene required for Drosophila olfaction. Genetics 124, 293-302.

Lin, J. Y., Knutsen, P. M., Muller, A., Kleinfeld, D., and Tsien, R. Y. (2013a). ReaChR: a red-shifted variant of channelrhodopsin enables deep transcranial optogenetic excitation. Nat. Neurosci. 16, 1499-1508. doi: 10.1038/nn.3502

Lin, J. Y., Sann, S. B., Zhou, K., Nabavi, S., Proulx, C. D., Malinow, R., et al. (2013b). Optogenetic inhibition of synaptic release with chromophore-assisted light inactivation (CALI). Neuron 79, 241-253. doi: 10.1016/j.neuron.2013.05.022

Liu, C., Placais, P. Y., Yamagata, N., Pfeiffer, B. D., Aso, Y., Friedrich, A. B., et al. (2012). A subset of dopamine neurons signals reward for odour memory in Drosophila. Nature 488, 512-516. doi: 10.1038/nature11304

Michels, B., Diegelmann, S., Tanimoto, H., Schwenkert, I., Buchner, E., and Gerber, B. (2005). A role for Synapsin in associative learning: the Drosophila larva as a study case. Learn. Mem. 12, 224-231. doi: 10.1101/lm.92805
Monte, P., Woodard, C., Ayer, R., Lilly, M., Sun, H., and Carlson, J. (1989). Characterization of the larval olfactory response in Drosophila and its genetic basis. Behav. Genet. 19, 267-283. doi: 10.1007/BF01065910

Nagel, G., Brauner, M., Liewald, J. F., Adeishvili, N., Bamberg, E., and Gottschalk, A. (2005). Light activation of channelrhodopsin-2 in excitable cells of Caenorhabditis elegans triggers rapid behavioral responses. Curr. Biol. 15, 2279-2284. doi: 10.1016/j.cub.2005.11.032

Pauls, D., Pfitzenmaier, J. E., Krebs-Wheaton, R., Selcho, M., Stocker, R. F., and Thum, A. S. (2010). Electric shock-induced associative olfactory learning in Drosophila larvae. Chem. Senses 35, 335-346. doi: 10.1093/chemse/bjq023

Rodrigues, V. (1980). Olfactory behavior of Drosophila melanogaster. Basic Life Sci. 16, 361-371. doi: 10.1007/978-1-4684-7968-3_26

Rohwedder, A., Wenz, N. L., Stehle, B., Huser, A., Yamagata, N., Zlatic, M., et al. (2016). Four individually identified paired dopamine neurons signal reward in larval Drosophila. Curr. Biol. 26, 661-669. doi: 10.1016/j.cub.2016.01.012

Saraswati, S., Fox, L. E., Soll, D. R., and Wu, C. F. (2004). Tyramine and octopamine have opposite effects on the locomotion of Drosophila larvae. J. Neurobiol. 58, 425-441. doi: 10.1002/neu.10298

Scherer, S., Stocker, R. F., and Gerber, B. (2003). Olfactory learning in individually assayed Drosophila larvae. Learn. Mem. 10, 217-225. doi: 10.1101/lm.57903

Schroll, C., Riemensperger, T., Bucher, D., Ehmer, J., Voller, T., Erbguth, K., et al. (2006). Light-induced activation of distinct modulatory neurons triggers appetitive or aversive learning in Drosophila larvae. Curr. Biol. 16, 1741-1747. doi: 10.1016/j.cub.2006.07.023

Schwaerzel, M., Monastirioti, M., Scholz, H., Friggi-Grelin, F., Birman, S., and Heisenberg, M. (2003). Dopamine and octopamine differentiate between aversive and appetitive olfactory memories in Drosophila. J. Neurosci. 23, 10495-10502.

Selcho, M., Pauls, D., El Jundi, B., Stocker, R. F., and Thum, A. S. (2012). The role of octopamine and tyramine in Drosophila larval locomotion. J. Comp. Neurol. 520, 3764-3785. doi: 10.1002/cne.23152

Selcho, M., Pauls, D., Han, K. A., Stocker, R. F., and Thum, A. S. (2009). The role of dopamine in Drosophila larval classical olfactory conditioning. PLoS ONE 4:e5897. doi: 10.1371/journal.pone.0005897

Selcho, M., Pauls, D., Huser, A., Stocker, R. F., and Thum, A. S. (2014). Characterization of the octopaminergic and tyraminergic neurons in the central brain of Drosophila larvae. J. Comp. Neurol. 522, 3485-3500. doi: $10.1002 /$ cne. 23616

Störtkuhl, K. F., and Fiala, A. (2011). The smell of blue light: a new approach toward understanding an olfactory neuronal network. Front. Neurosci. 5:72. doi: 10.3389/fnins.2011.00072

Tracey, W. D. Jr., Wilson, R. I., Laurent, G., and Benzer, S. (2003). Painless, a Drosophila gene essential for nociception. Cell 113, 261-273. doi: 10.1016/S0092-8674(03)00272-1

Tully, T., Cambiazo, V., and Kruse, L. (1994). Memory through metamorphosis in normal and mutant Drosophila. J. Neurosci. 14, 68-74.

Vosshall, L. B., and Stocker, R. F. (2007). Molecular architecture of smell and taste in Drosophila. Annu. Rev. Neurosci. 30, 505-533. doi: 10.1146/annurev.neuro.30.051606.094306

Wang, J. W., Wong, A. M., Flores, J., Vosshall, L. B., and Axel, R. (2003). Twophoton calcium imaging reveals an odor-evoked map of activity in the fly brain. Cell 112, 271-282. doi: 10.1016/S0092-8674(03)00004-7

Zhang, T., Branch, A., and Shen, P. (2013). Octopamine-mediated circuit mechanism underlying controlled appetite for palatable food in Drosophila. Proc. Natl. Acad. Sci. U.S.A. 110, 15431-15436. doi: 10.1073/pnas.1308816110

Conflict of Interest Statement: The authors declare that the research was conducted in the absence of any commercial or financial relationships that could be construed as a potential conflict of interest.

Copyright (c) 2016 Honda, Lee, Honjo and Furukubo-Tokunaga. This is an openaccess article distributed under the terms of the Creative Commons Attribution License (CC BY). The use, distribution or reproduction in other forums is permitted, provided the original author(s) or licensor are credited and that the original publication in this journal is cited, in accordance with accepted academic practice. No use, distribution or reproduction is permitted which does not comply with these terms. 